\title{
Oviposition of the Fir Sawyer Beetle (Monochamus urussovi Fisch.) on the Tree Stem: Analysis Using Models of Random Point Fields
}

\author{
Olga P. Sekretenko ${ }^{\mathrm{a}, \mathrm{b} *}$, \\ Anton V. Kovalev ${ }^{\mathrm{b}}$ and Vladislav G. Soukhovolsky ${ }^{\mathrm{a}, \mathrm{b}}$ \\ a Sukachev Institute of Forest SB RAS \\ 50/28 Akademgorodok, Krasnoyarsk, 660036, Russia \\ ${ }^{b}$ Krasnoyarsk Sceintific Centre SB RAS \\ 50 Akademgorodok, Krasnoyarsk, 660036, Russia
}

Received 28.04.2013, received in revised form 21.12.2013, accepted 07.09.2014

The fir sawyer beetle is a xylophagous species that develops outbreaks in boreal coniferous forests. Females deposit single eggs under the bark through slotted holes that they make by their mandibles. These oviposition scars are clearly visible while the tree is alive. Larvae feed on phloem and sapwood for one or two summers. Under the bark each larva forms compact damage area close to the oviposition scar. One larva needs an area of no less than $50 \mathrm{sq} \mathrm{cm}$ for feeding. In this study, we tested the hypothesis that adult females optimize the eggs' spatial pattern to reduce the possibility that the feed zones of expectant larvae will overlap one another. This oviposition strategy reduces larvae competition and enhances their survival. During the outbreak's peak phase the stems of damaged fir trees were photographed and the coordinates of the 317 oviposition scars were recorded. Spatial statistics was used to determine that the distribution of eggs on the stem surface is regularized: close to the oviposition scar the number of another scars are less than this number for the independent distribution of points. The point process model with negative interacion between points was fitted to the oviposition locations data. The force of the repulsive interaction was found diminishing with increasing of the distance between the scars. The radius of interaction was estimated to be $4 \mathrm{~cm}$. Therefore, the reproductive strategy of the fir sawyer beetle includes oviposition behavior aimed to minimize larval competition.

Keywords: Monochamus urussovi Fisch., oviposition behaviour, food competition, reproductive strategy, plant-insect interaction, spatial point process, point pattern, spatial statistics.

(c) Siberian Federal University. All rights reserved

* Corresponding author E-mail address: sekretenko@ksc.krasn.ru 


\title{
Размещение яиц черного пихтового усача \\ (Monochamus urussovi Fisch.) на стволе дерева: \\ анализ с использованием моделей \\ случайных точечных полей
}

\author{
О.П. Секретенко ${ }^{\mathrm{a}, \boldsymbol{\sigma}}$, \\ А.В. Ковалев ${ }^{\tilde{0}}$, В.Г. Суховольский ${ }^{\mathrm{a}, \tilde{0}}$ \\ ${ }^{a}$ Институт леса им. В.Н. Сукачева СО РАН \\ Россия, 660036, Красноярск, Академгородок, 50/28 \\ ${ }^{6}$ Международный научный иентр исследования \\ экстремальных состояний организма при Президиуме КНЦ СО РАН \\ Россия, 660036, Красноярск, Академгородок, 50
}

Черный пихтовый усач - ксилофаг, образующий в темнохвойных лесах очаги массового размножения. Жуки при заселении дерева оставляют на стволе хорошо видимые насечки отверстия, каждое из которых используется для помещения под кору одного яйца. Личинка в течение следующего лета питается лубом, образуя под корой непосредственно около насечки компактное повреждение. Для развития личинки необходима площадь питания не менее $50 \mathrm{~cm}^{2}$. В настоящей работе проверено предположение о том, что самки размещают яйца так, чтобы уменьшить возможность перекрытия зон питания личинок. В очаге массового размножения по фотографиям поврежденных стволов были определены координаты 317 насечек. Методами пространственной статистики установлено, что размещение насечек является регуляризованным: в ближайшей окрестности каждой из них, как правило, количество других насечек значимо меньше, чем было бы при их независимом распределении. Размещение насечек описано моделью случайного точечного поля с негативным взаимодействием между его элементами. Показано, что интенсивность «расталкивающего взаимодействия» тем больше, чем меньше дистанциия между насечками. Радиус взаимодействия составляет 4 см. Полученные результаты служат свидетельством в пользу того, что репродуктивная стратегия черного пихтового усача включает оптимизацию пространственного размещения потомства на стадии откладки яии для уменьшения конкуренщии за корм между будущими личинками.

Ключевые слова: репродуктивная стратегия, конкуренция за корм, взаимодействие растений и насекомых, случайное точечное поле, пространственная статистика.

\section{Введение}

Репродуктивное поведение многих видов насекомых включает в себя заботу родительского поколения о потомстве (Мозолевская и др., 2010). Не только семьи и колонии общественных насекомых (муравьи, пчелы, осы, термиты), но также самки и брачные пары некоторых других видов (уховертки, жуки-могильщики, одиночные осы и пчелы) ухаживают за своими отпрысками, оберегая 
отложенные яйца и выкармливая личинок. Литература по этой теме безгранична. Однако для многих видов насекомых с неперекрывающимися поколениями, у которых имаго и личинки никогда не встречаются, забота о потомстве также является составной частью наследственной репродуктивной программы (Janz, 2002; Мозолевская и др., 2010). Шансы потомства на выживание зависят от множества факторов: количества и качества доступного корма, защищенности от хищников, паразитов, болезней, абиотических факторов. Реакция на каждый из этих факторов может отражаться в выборе места для откладки яиц, устройстве укрытий, обеспечении запаса корма для личинок (Randlkofer et al., 2007; Sadek et al., 2010; Павлов, 2011; Castillo et al., 2013). Если на личиночной стадии перемещения особей ограничены и сменить кормовой объект для них невозможно, то выбор самкой места размещения яйца однозначно определяет объем и качество корма, доступного потомку.

Внутривидовая конкуренция за кормовые ресурсы для потомства присуща насекомымксилофагам, у которых самка откладывает яйца под кору дерева, а вышедшие из яиц личинки следующего поколения питаются лубом дерева, выбранного родителем (Мозолевская и др., 2010). Если считать, что самки ксилофагов размещают яйца на таком расстоянии одно от другого, которое обеспечивает каждой из будущих личинок необходимую площадь питания, то такое поведение было бы оптимальным с точки зрения выживания потомства, если бы не было других плотностнозависимых факторов, также влияющих на его выживаемость. Тем не менее равномерность размещения яиц на разных субстратах, интерпретируемая как результат действия инстинкта, направленного на уменьшение конкуренции личинок, отмечается во множестве работ (Исаев и др., 1988; Janz, 2002;
Leyva et al., 2003; Holland et al., 2004; Horn, Holland, 2010; Castillo et al., 2013).

Еще одной стороной взаимодействия насекомых выступает совместное преодоление устойчивости растения к их повреждающему воздействию. В процессе питания насекомые-фитофаги сталкиваются с рядом иммуногенетических защитных систем растений - тканевыми, осмотическими, физиологическими барьерами (Мозолевская и др., 2010). Токсичные соединения, вырабатываемые растением в ответ на повреждение, убивают насекомых или существенно затрудняют их питание (Исаев, Гирс, 1975; Lempa et al., 2004; Бахвалов и др., 2010). Устойчивость растений к повреждению является одним из важных факторов, определяющих динамику численности насекомых (Исаев и др., 2001; Бахвалов и др., 2010). Реакция дерева может быть как генерализованной, так и локальной, затрагивающей только определенный объем тканей (Arimura et al., 2004). После некоторого критического повреждающего воздействия насекомых негативная ответная реакция в какой-либо части растения может снижаться в связи с истощением защитных ресурсов (Herms, Mattson, 1992). Так, черный пихтовый усач на начальном этапе заселения дерева откладывает яйца не по всему стволу, а сосредотачивая их в определенной области (у комля или в средней части ствола, в зависимости от условий). При этом значительная доля личинок первого года поселения гибнет в ходе преодоления «смоляного барьера», а на следующий год, когда дерево уже ослаблено заселившимися ранее личинками, выживаемость становится больше, хотя роль питания жуков побегами кроны в ослаблении дерева более значительна (Исаев и др., 1988). Описаны также эксперименты с усачами вида Monochamus alternatus, в которых показано, что выживаемость одиночно инокулированных личинок меньше, 
чем размещенных парами (Anbutsu, Togashi, 1997). Установлено, что агрегированное поселение насекомых-фитофагов во многих случаях служит проявлением их кооперативного взаимодействия для локального преодоления защитной реакции растения (Clark, Faeth, 1997; Denno, Benrey, 1997; Desurmont, Weston, 2011).

Можно ожидать, что у насекомых процесс откладки яиц включает некоторую «стратегию» по оптимизации плотности и характера расположения личинок на кормовом растении, учитывающую конкуренцию за корм и кооперацию по преодолению энтоморезистентности дерева. Взаимодействия между особями, необходимые для реализации такой стратегии, обеспечиваются нанесением метки - особого феромона - в места, куда были помещены яйца (Anbustu, Togashi, 2000; Janz, 2002; Peddle et al., 2002; Мозолевская и др, 2010; Horn, Holland, 2010). Например, описано, что жуки рода Monochamus перед размещением очередного яйца тщательно обследуют поверхность ствола и насечки других самок, чтобы обнаружить и учесть положение уже отложенных яиц и развивающихся личинок (Anbutsu, Togashi, 2000; Peddle et al., 2002). Руководствуются они при этом запахом специального секрета, которым были запечатаны входы в яйцевые камеры, устроенные самками под корой.

Статистические исследования для обнаружения проявлений конкуренции за корм чаще всего проводятся в случаях, когда насекомые заселяют дискретный субстрат, например, венчики цветов, плоды, семена (Holland et al., 2004; Paukku, Kotiaho, 2008; Horn, Holland, 2010; Castillo et al., 2013). Koличественное изучение разделения общего кормового ресурса между многими особями встречается реже. В нашей работе взаимодействие насекомых между собой и с кор- мовым объектом анализируется на примере черного пихтового усача - очень удобного объекта для таких исследований. Жуки этого вида в точке откладки каждого яйца оставляют на коре дерева хорошо видимый след, так называемую насечку (Исаев и др., 1988). Появляющиеся личинки не устраивают под корой длинных ходов, как это бывает у многих ксилофагов, а выедают в лубе компактную площадку вблизи насечки. Россыпь насечек образует на поверхности ствола некий рисунок - определенную пространственную конфигурацию точек, структура которой может отражать поведенческие реакции самок по размещению потомства.

Пространственная статистика - раздел математической статистики, включающий модели и методы для изучения явлений, в которых пространственная компонента играет существенную роль. Объединяет три направления, различающиеся типом данных: геостатистику, предназначенную для описания непрерывно изменяющихся величин, например свойств почвенного покрова; пространственные точечные процессы (поля), характеризующие распределение дискретных объектов, например деревьев в лесу; модели для описания случайных мозаик и решеточных структур (Gelfand et al., 2010).

Пространственная структура биологических сообществ исследуется с середины прошлого века, методы пространственных точечных процессов используются для этого с 70-х годов (Gelfand et al., 2010). Чаще всего изучается горизонтальная структура растительных ценозов (например, Sekretenko, Gavrikov, 1998; Кузьмичев, Секретенко, 2001; Perry et al., 2006; Курбанов, Воробьев, 2008; Фардеева и др., 2009; Грабарник, 2010; Ухваткина, Омелько, 2011; Urea-Dies et al., 2013), однако популяции животных, в том числе насекомых, также являются объектами исследования (Harkness, 
Isham, 1983; Секретенко и др., 2002; Rossi et al., 2009; Grohmann et al., 2010).

Целью настоящей работы был количественный анализ особенностей репродуктивного поведения ксилофагов. Предполагалось, что оптимизация размещения состоит в том, что самки откладывают яйца достаточно далеко одно от другого для уменьшения конкуренции будущих личинок за корм, но при этом их локальная плотность достаточно велика для преодоления защитной реакции дерева. Методами пространственной статистики было проверено, согласуются ли предположения об оптимизации размещения потомства родительским поколением с характеристиками наблюдаемого расположения насечек на стволах пихты.

\section{Материалы и методы}

Черный пихтовый усач

В качестве объекта для пространственного анализа репродуктивного поведения ксилофагов был выбран черный пихтовый усач Monochamus urussovi Fisch. Этот вид питается на хвойных породах деревьев, часто образуя в пихтарниках очаги массового размножения (Исаев и др. 1988). При низкой численности жуки откладывают яйца под кору на необратимо ослабленных деревьях и отдельных поврежденных участках стволов живых деревьев. Поскольку жуки этого вида кормятся в кронах корой побегов, то при высокой плотности популяции они ослабляют здоровые деревья, делая их пригодными для откладки яиц и питания личинок. В условиях Сибири средняя плодовитость самки составляет 28 яиц за лето. Обычно откладывается по 3-5 штук в день с интервалом от 15 мин до 1.5 ч. Самки помещают яйца под кору по одному, выгрызая для каждого из них насечку - щелевидное углубление длиной 5-9 мм. Насечки сохраняются продолжительное вре- мя. Появившаяся из яйца личинка в течение одного-двух летних сезонов питается под корой вблизи насечки и образует неправильной формы компактную площадку, выедая около $50 \mathrm{~cm}^{2}$ тканей луба и поверхностного слоя заболони. Защитная реакция тканей дерева, выражающаяся в засмолении яиц и личинок, приводит к гибели значительной их части. Однако при массовом заселении ствола реакция дерева ослабляется и смертность насекомых на стадиях яйца и личинки уменьшается (Исаев и др., 1988).

\section{Сбор данныхх}

Данные собраны в Тасеевском районе Красноярского края в древостое, сформированном пихтой сибирской Abies Sibirica Ledeb. В очаге массового размножения черного пихтового усача сделаны цифровые фотографии участков стволов 11 поврежденных усачом деревьев с диаметрами 12-41 см. Координаты 317 насечек определены на развертках боковых поверхностей стволов с компенсацией искажений, возникших из-за цилиндричности стволов и геометрической аберрации, вносимой фотокамерой. Затененные части изображений, на которых насечки плохо видны, исключались из анализа. Учетные площадки представляли собой многоугольники величиной от 3 до 13 дм² (рис. 1, табл. 1).

\section{Статистический анализ}

Изучение закономерностей расположения насечек на развертках боковых поверхностей стволов выполняли методами, разработанными для анализа пространственных точечных структур с использованием моделей точечных полей, или процессов (Stoyan, Stoyan, 1994; Baddeley et al., 2006; Møller, Waagepetersen, 2007; Грабарник, 2010; Gelfand et al., 2010). «Точечные процессы, или случайные точечные поля, суть математические модели для 


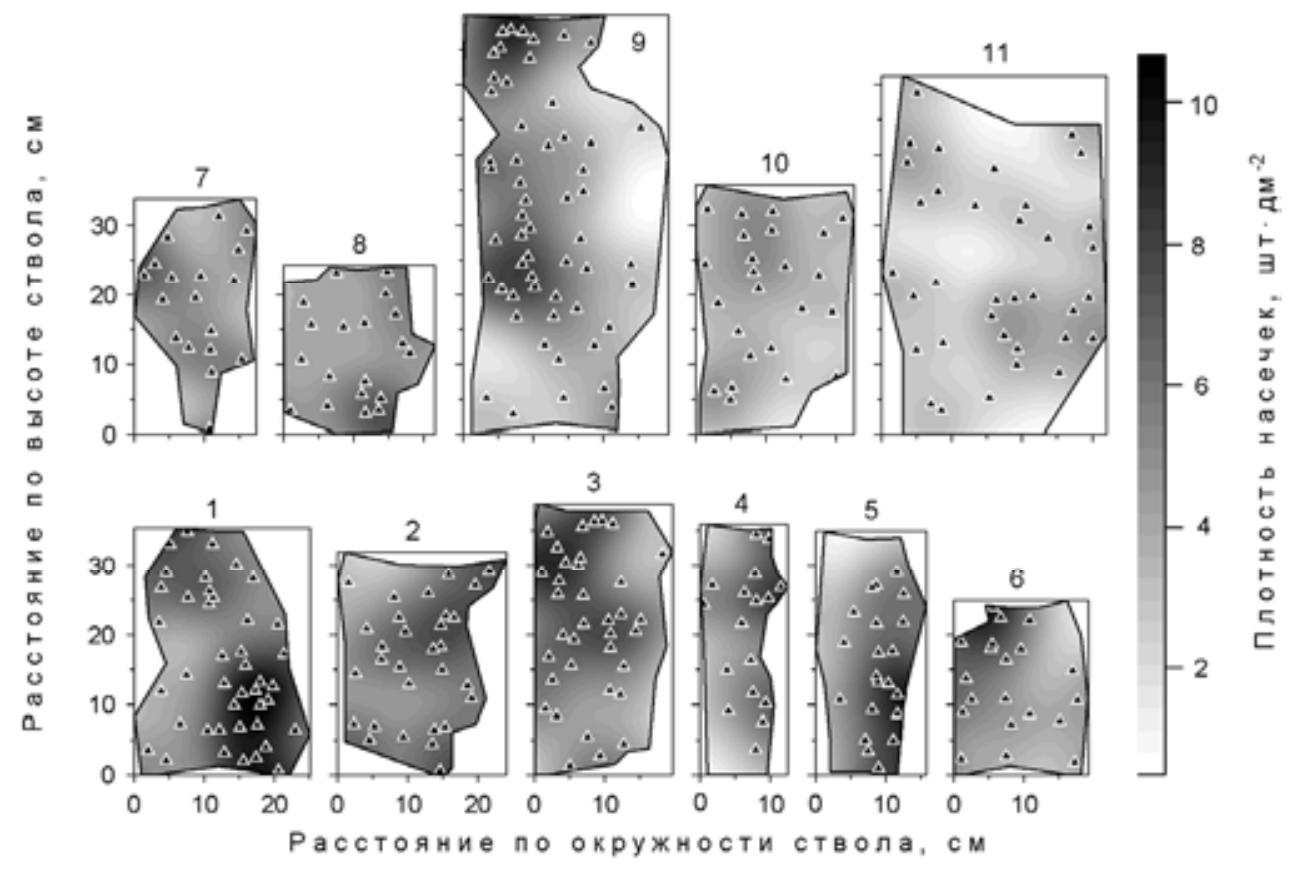

Рис. 1. Схема расположения насечек и их сглаженная плотность в пределах учетных площадок $(1-11)$ на развертках боковых поверхностей стволов деревьев

Таблица 1. Характеристики учетных площадок и их сравнение по плотности насечек

\begin{tabular}{c|c|c|c}
\hline Номер дерева & Диаметр ствола, см & Число насечек, шт. & $\begin{array}{c}\text { Плотность насечек, } \\
\text { шт:дм }\end{array}$ \\
\hline 1 & 24 & 42 & $6.3^{*}$ \\
2 & 29 & 30 & $5.7^{*}$ \\
3 & 27 & 36 & $5.6^{*}$ \\
4 & 12 & 17 & 5.0 \\
5 & 19 & 21 & 5.0 \\
6 & 18 & 20 & 4.9 \\
7 & 28 & 18 & 4.8 \\
9 & 19 & 19 & 4.7 \\
10 & 41 & 55 & 4.1 \\
11 & 29 & 24 & 3.6 \\
\hline
\end{tabular}

*Площадка на дереве 11 отличается по плотности насечек от площадок на деревьях 1, 2 и 3 на уровне значимости $p<0.05$.

дискретных множеств точек, случайно распределенных в пространстве» (Амбарцумян и др., 1989, с. 305). Основные понятия, связанные с используемыми в настоящей работе моделями, описаны в прил. А, а конкретные применяемые статистические методы - в прил. Б со ссылками на соответствующие литературные источники. Расчеты проводили в 
свободно распространяемой статистической среде R (R Core Team, 2013), которая включает язык программирования $\mathrm{R}$ и полный набор функций для любых «традиционных» статистических вычислений, а также множество специализированных пакетов, создаваемых учеными-статистиками для реализации и представления новых методов по мере их разработки. Нами использовался пакет spatstat, предназначенный для всестороннего исследования пространственных точечных структур (Baddeley, Turner, 2005).

Для предварительной диагностики размещения насечек были рассчитаны две характеристики - сглаженная функияия интенсивности (плотности), описывающая, как меняется число насечек на единице площади в масштабах, сопоставимых с размером учетной площадки, а также парная корреляичонная функция, характеризующая взаимное расположение насечек относительно друг друга (прил. Б). Далее последовательно проверяли предположения о свойствах размещения насечек, отражающих поведение самок усача во время откладки яиц, сформулированные в виде статистических моделей точечных noлей. Исследованы четыре комбинации предположений: 1) поверхность ствола в отношении выживаемости личинок однородна и взаимодействия самок нет - однородное пуассоновское поле; 2) поверхность неоднородна, взаимодействия самок также нет - неоднородное пуассоновское поле; 3) поверхность однородна, взаимодействие есть - однородное поле Гиббса; 4) поверхность неоднородна, взаимодействие есть - неоднородное поле Гиббса (прил. А). Параметры моделей были оценены по данным наблюдений для каждого из этих четырех вариантов методом псевдоправдоподобия (прил. Б). Гипотезы согласия моделей с натурными данными тестировались методом огибающих (прил. Б). Если модель признава- ли не соответствующей данным, считали, что предполагаемые характеристики поведения самок не соответствуют действительности.

\section{Результаты}

Плотность насечек

Средние по каждой из учетных площадок плотности насечек лежат в интервале от 2.8 до 6.3 шт дм ${ }^{-2}$, т.е. различаются более чем в два раза (табл. 1). Если считать распределение насечек в пределах каждой площадки независимым и однородным и проверить различие плотностей насечек на значимость (Кокс, Льюис, 1969), то оказывается, что на уровне значимости 0.05 только площадка с минимальной плотностью насечек отличается от каждой из трех площадок, на которых плотность 5.6 шт·дм ${ }^{-2}$ и выше. Все остальные пары площадок между собой значимо не различаются. Коэффициент корреляции (по Пирсону) между плотностью насечек на дереве и его диаметром равен -0.46. При числе наблюдений 11 эта величина отличается от нуля только на уровне значимости 0.15. Следовательно, связи плотности насечек с диаметром дерева не обнаруживается.

На рис. 1 вместе с расположением насечек представлена их двумерная функиия интенсивности (плотности), рассчитанная путем сглаживания функцией Гаусса (прил. Б) со стандартным отклонением 4 см. Известно, что для питания одной личинки черного пихтового усача необходима площадь около

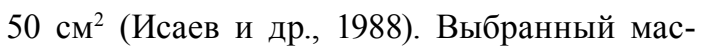
штаб сглаживания примерно соответствует радиусу площадки такого размера $\left(\pi \cdot 4^{2}=50.3\right)$. Можно предполагать, что плотность насечек за пределами этого расстояния слабо влияет на выбор самкой конкретной точки поверхности в качестве места откладки яйца. При таком способе сглаживания значение плотности, соотнесенное с данной точкой поверхно- 
сти, приблизительно на 70 \% зависит от числа насечек, находящихся от нее на расстоянии до 4 см. Значения сглаженной плотности насечек в разных частях площадок варьируют от 0.2 до 11 шт-дм². Если считать, что самка при откладке яйца учитывает положение уже существующих насечек и свойства субстрата преимущественно в области такого размера, который необходим для развития личинки, то наблюдаемую изменчивость плотности насечек вряд ли можно рассматривать как несущественную.

\section{Модели независимого}

размещения насечек

Для предварительного анализа размещения насечек рассчитана эмпирическая парная коррелящионная функиия, характеризующая частоту встречаемости пар насечек в зависимости от расстояния между ними (прил. Б). В случае однородного пуассоновского точечного поля, являющегося классической моделью для описания стохастического распределения невзаимодействующих точечных объектов по плоскости, свойства которой везде одинаковы в том смысле, что не влияют на их расположение, парная корреляционная функция равна единице для любых расстояний между объектами. Расчеты показали, что на расстояниях от 0 до 3 см значение парной корреляционной функции увеличивается от 0.2 до 1 , при больших расстояниях практически не изменяется (рис. 2), что позволяет говорить, что вблизи насечки, как правило, есть область, в пределах которой другие насечки встречаются достаточно редко, т.е. «взаимодействие» между ними имеет характер «расталкивания». Радиус этой области составляет приблизительно 3 см. На расстояниях, превышающих 3 см, пространственной корреляции между расположением насечек не прослеживается.

Поскольку не исключено, что такая парная корреляционная функция может соответствовать также случаю, когда взаимодействия между точками или однородности поверхности нет, методом статистического моделирования было выполнено тестирование гипотез о согласии моделей однородного и неоднородного точечного поля Пуассона с наблюдаемыми данными (рис. 2a, б). В первой моде-
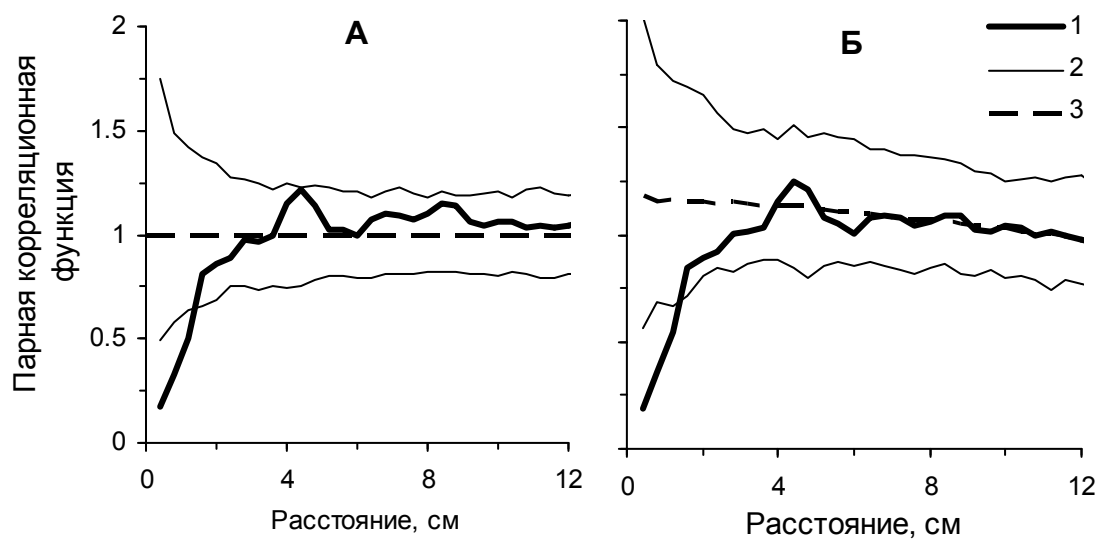

Рис. 2. Эмпирическая парная корреляционная функция распределения насечек (1) с двусторонними областями отклонения гипотезы о согласии моделей однородного (А) и неоднородного (Б) точечных полей Пуассона с натурными данными. 2 - границы верхних и нижних частей областей отклонения гипотезы о согласии моделей и данных, 3 - теоретические парные корреляционные функции моделей 
ли предполагали отсутствие взаимодействия между насечками и однородность поверхности стволов, вторая отличалась тем, что разные участки поверхности считались неодинаково подходящими для откладки яиц.

Гипотезы согласия моделей с натурными данными тестировали методом статистического моделирования. Границы двусторонней области отклонения гипотезы (огибающие) определены по 1500 реализациям соответствующих моделей так, что вероятность ошибки первого рода составила меньше 5 \% для всех используемых моделей. Область отклонения гипотезы состоит из двух частей, одна из которых находится над верхней огибающей, другая - под нижней. Если какие-либо значения парной корреляционной функции, рассчитанные по данным наблюдений, распологаются в пределах этой области, то гипотеза о согласии модели и данных отклоняется. Подробно метод тестирования описан в прил. Б.

Так как на расстояниях до 2 см значения парной корреляционной функции меньше нижней огибающей и в первом, и во втором случае (рис. 2a, б), то гипотезы согласия моделей пуассоновских точечных полей и натурных данных отклоняются. Таким образом, нельзя считать, что выбор самкой места для откладки яйца не зависит от расположения уже сделанных насечек. Предположение, что наблюдаемое распределение представляет собой группы «не взаимодействующих» между собой насечек, образовавшихся за счет неоднородности субстрата, также не является адекватным.

\section{Моделирование зависимости} взаимного размещчения насечек

Взаимодействие насечек понимается как зависимость их расположения относительно друг друга. Самки усачей в процессе откладки яиц, не контактируя непосредственно друг с другом, обследуют уже сделанные насечки и ориентируются на запаховые метки, оставленные в них другими самками сразу после откладки яйца (Исаев и др., 1998; Anbutsu, Togashi, 2000). Более подробная информация о типе и «силе взаимодействия» между насечками была получена с использованием моделей гиббсовских точечных полей (прил. А), для которых структура размещения точек поля описывается с помощью функции взаимодействия $h(r)$, задающей изменение с расстоянием $r$ «силы» влияния точек друг на друга, отражающегося в их взаимном расположении. Гиббсовские точечные поля описываются с помощью условной интенсивности, которая определяет вероятность обнаружить точку поля в точке плоскости с определенными координатами, при условии, что положение всех остальных точек поля уже задано (Ripley, 1990). Для наблюдаемого размещения точечных объектов с координатами $\mathbf{X}=\left\{x_{i}, \ldots, x_{n}\right\}$, где $n$ - число точек, условная интенсивность $\lambda(u, \boldsymbol{X})$ в точке плоскости $u$ может быть представлена в виде произведения двух функций:

$$
\lambda(u, \boldsymbol{X})=B(\psi, u) \cdot C(\varphi, u, \boldsymbol{X})
$$

где $B(\psi, u)$ - функция, описывающая изменение интенсивности по плоскости; $C(\varphi, u, \boldsymbol{X})$ - функция, описывающая стохастическое взаимодействие между точками поля; $\psi, \varphi-$ параметры. Функция $C(\varphi, u, \boldsymbol{X})$ представляет собой произведение значений функций взаимодействия всех точек поля в точке плоскости $u$ :

$$
C(\varphi, u, \mathbf{X})=\prod_{\substack{i=1 \\ x_{i} \neq u}}^{n} h\left(\varphi, u, x_{i}\right)
$$

где $h\left(\varphi, u, x_{i}\right)$ - функция взаимодействия, описывающая влияние $i$-й точки поля. Обычно она задается так, чтобы обозначить, что 
если расстояние между двумя точками поля больше некоторой пороговой величины, то взаимодействия между ними нет. Значения функции больше единицы соответствуют «притяжению», меньше единицы - «расталкиванию» точек поля.

При рассмотрении расположения насечек условная интенсивность может интерпретироваться как привлекательность точки поверхности для откладки очередного яйца, зависящая от свойств поверхности ствола в данной точке и от положения всех уже имеющихся насечек и их «взаимодействия». Под «взаимодействием» насечек имеется в виду, что их расположение есть результат взаимодействия самок, направленного на обеспечение наибольшей выживаемости их потомства.

Поскольку данные, используемые в этой работе, не содержали информации о том, однородна ли поверхность стволов по толщине и качеству луба и коры в пределах учетных площадок, рассматривали две равноправные модели Гиббса, описывающие расположение насечек с учетом взаимодействия между ними.

В первом случае предполагалось, что самки не выделяют какие-либо области на стволе или что отдельные области ствола равноценны с точки зрения выживаемости личинок. Тогда итоговый рисунок распределения насечек - это результат одного только взаимодействия самок, а агрегированность насечек есть результат прогнозирования самкой кооперативного взаимодействия будущих личинок по преодолению устойчивости дерева к повреждению. В этом случае функция $B(\psi, u)$ не зависит от координаты $u$ и является константой. Условная интенсивность в этой модели определяется только функцией взаимодействия. Предварительные расчеты показали, что за пределами расстояния в 4 см взаимодействие между насечками исчезает, парная корреляционная функция (рис. 2) свидетельствует об этом же. Функция взаимодействия, которая характеризует зависимость силы взаимодействия между двумя насечками от расстояния между ними, была задана в виде ступенчатой функции для значений расстояний от 0 до 4 см с шагом 0.5 см:

$$
h(r)=\left\{\begin{array}{cc}
\varphi_{1} & r \leq 0.5 c M \\
\varphi_{2} & 0.5 c M<r \leq 1 c M \\
\cdots & \\
\varphi_{8} & 3.5 c M<r \leq 4 c M \\
1 & r>4 c M
\end{array}\right.
$$

Здесь $\varphi_{1}, \ldots, \varphi_{8}$ - числовые параметры функции взаимодействия, которые рассчитываются на основе имеющихся данных в результате «фитирования» модели.

Вторая модель соответствует предположению, что причиной некоторой агрегированности насечек является неоднородность свойств ресурса - поверхности ствола дерева. Известно, что жуки заселяют в первую очередь места вокруг трещин, подсушин на южной стороне ствола, т.е. могут оценить качество корма (Исаев и др., 1988). Тогда пространственный рисунок расположения насечек складывается из совместного влияния вида распределения ресурса и «взаимодействия» насечек. В данной модели предполагалось также, что если поверхность различается по свойствам, то самки при откладке яиц уже учли это различие. В этом случае наблюдаемая повышенная локальная плотность насечек в некоторых частях учетных площадок рассматривается как количественный показатель большей привлекательности этих частей стволов для размещения яиц. Детерминированная компонента модели тогда пропорциональна уже определенной выше локальной плотности насечек, численно заданной 
в каждой точке $u$ учетных площадок. Второй сомножитель, как и в предыдущем варианте, задан произведением парных функций взаимодействия между насечками. Отличие этой модели от предыдущей состоит в том, что детерминированная компонента здесь зависит от координат. Функция взаимодействия задана, как и для первого случая, в ступенчатом виде, однако рассчитанные значения высот ступенек будут в этой модели другими.

Параметры модели были оценены методом максимального псевдоправдоподобия (Baddeley, Turner, 2000) по натурным данным - координатам насечек, снятых с фотографий стволов. В первой модели одновременно оценивалось восемь параметров, представляющих собой значения высот ступенек функции взаимодействия (формула 2). Во второй модели к оцениваемым параметрам добавлен еще коэффициент $\psi$, характеризующий влияние неравномерно распределенного peсурса. Функции взаимодействия заданы парные и изотропные, учитывающие силу и направленность взаимодействия только пар насечек и не зависящие от ориентации пар в пространстве.

Для двух моделей функции взаимодействия, оцененные по одним и тем же данным, получились различными в зависимости от того, считается ли ресурс однородным. В первом случае значения функции взаимодействия на расстояниях от 0 до 2 см меньше единицы, что означает «расталкивание» между насечками. На расстояниях же от 2 до 4 см значения функции взаимодействия больше единицы, что соответствует положительному взаимодействию, т.е. некоторому «притягиванию» (рис. 3a). Таким образом, после откладки яйца в непосредственной близости к нему образуется зона, неблагоприятная для откладки следующих яиц из-за конкуренции за корм, а на расстоянии 2-4 см - более бла- гоприятная, чем на расстояниях за пределами 4 см. Предполагается, что ресурс распределен однородно (рис. 3б). Если это предположение справедливо, то такая модель трактует наблюдаемое расположение насечек в пользу существования поведенческих реакций самок, направленных на одновременный учет конкуренции между личинками за корм и кооперации между ними для возможного ослабления ингибирующего влияния дерева. Тогда самкам «выгодно» располагать яйца, разделяя их некоторым расстоянием для обеспечения питания, но не слишком далеко друг от друга, чтобы использовать преимущества совместного поселения для преодоления ответной защитной реакции дерева. При этом образуются группы с расталкиванием отдельных элементов внутри них.

Во второй модели на расстояниях от 0 до 5 см значения функции взаимодействия постепенно увеличиваются от 0 до 1 (рис. 3г), что означает негативное взаимодействие, с постепенным ослаблением силы взаимодействия с расстоянием. Оцененный коэффициент $\psi$, характеризующий вклад ресурса, неоднородно распределенного по поверхности ствола (рис. 3д), равен 0.56, и, поскольку он значимо отличен от нуля и положителен, это означает возможность влияния гипотетической негомогенности ресурса. Такая модель интерпретирует характеристики наблюдаемого расположения насечек на стволах дерева как учет самкой усача в процессе откладки яиц конкуренции личинок за неоднородный pecypc.

Прогноз возможности дальнейшего заселения для любой точки поверхности может быть сделан при помощи моделей с оцененными параметрами при условии, что заданы координаты всех уже существующих насечек. Условная интенсивность точечного поля может интерпретировать- 
Модель 1: конкуренция-кооперация при однородном ресурсе

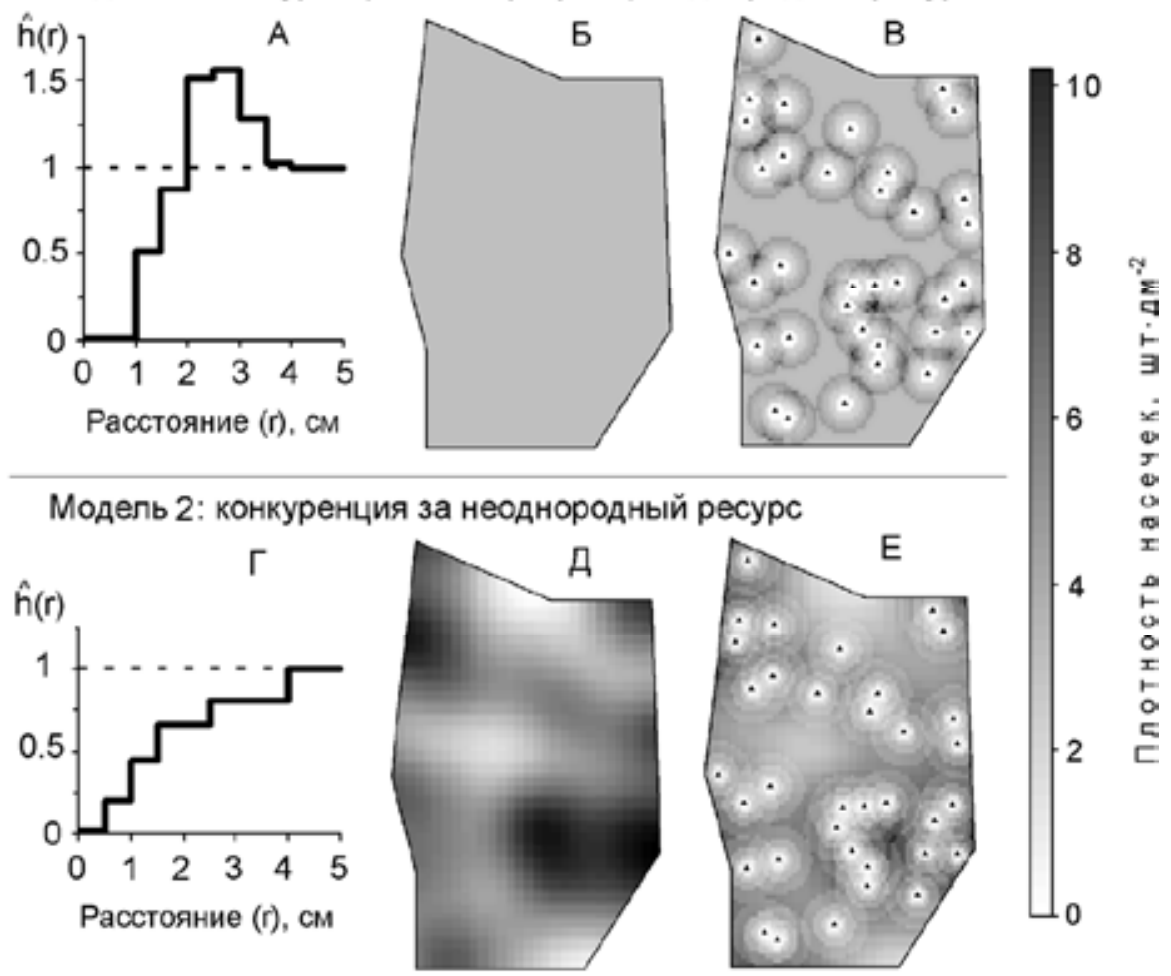

Рис. 3. Функции взаимодействия $\hat{h}(r)$ точечных полей, оцененные по всему набору данных (А, Г),

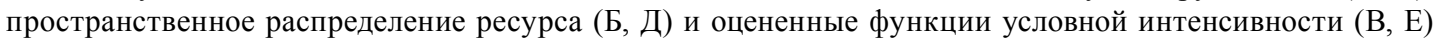
на примере учетной площадки № 11 в моделях точечных полей Гиббса с однородным и неоднородным распределением ресурса соответственно

ся как относительная привлекательность каждого конкретного небольшого участка ствола для откладки очередного яйца при учете положения всех других уже отложенных яиц и известной (или неизвестной, как во второй модели) неоднородности свойств самой поверхности ствола, не зависящей от насечек. Условная интенсивность была оценена по данным наблюдений и моделям как функция координат на решетке с шагом 0.25 см (рис. 3в, е). Области с низкими ее значениями в данном случае должны рассматриваться как наименее пригодные для откладки следующего дополнительного яйца при существующей конфигурации в расположении уже отложенных яиц, а области с высокими значениями - как наиболее привлекательные. Для первой модели (модель «конкуренция-кооперация»), несмотря на однородность ресурса, вблизи групп насечек просматриваются благоприятные для поселения области, образующиеся за счет того, что взаимодействие насечек на расстояниях 2-4 см положительно (рис. 3в), что может быть проинтерпретировано как учет самкой кооперативного взаимодействия личинок по преодолению негативной реакции дерева на повреждение. Для второй модели (модель «конкуренция за неоднородный ресурс») на рис. 3е видно, что в областях с «хорошим» ресурсом имеются участки низкой привлекательности для дальнейшего поселения из-за конкуренции, так как они уже частично заняты насечками. 

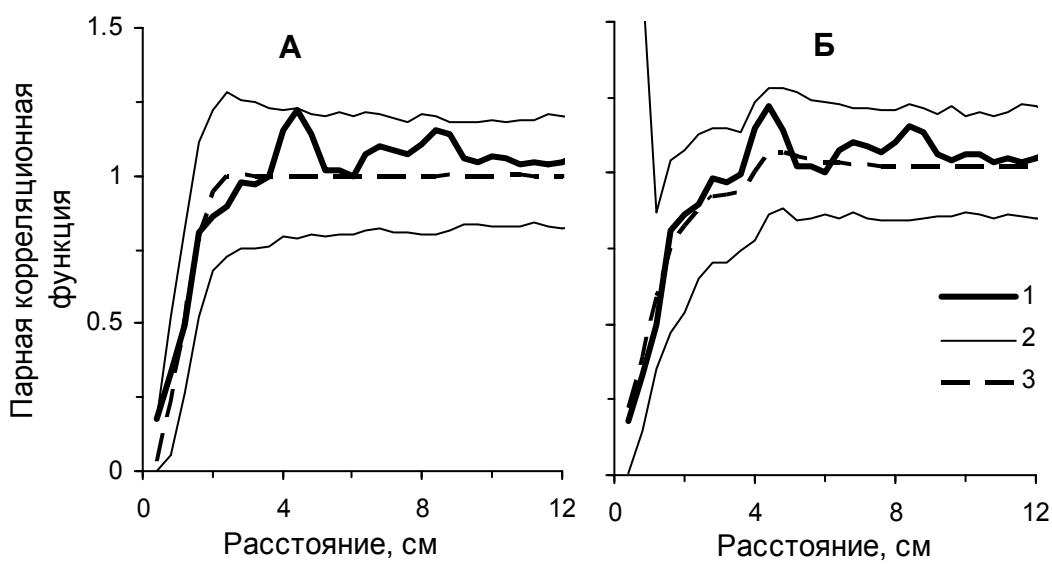

Рис. 4. Эмпирическая парная корреляционная функция распределения насечек (1); верхние и нижние границы двусторонних областей отклонения гипотез о согласии моделей с натурными данными для однородного (А) и неоднородного (Б) точечных полей Гиббса (2); парные корреляционные функции, усредненные по реализациям моделей (3)

Проверка гипотез о согласии этих двух моделей с данными была выполнена тем же методом, что и для пуассоновских моделей (прил. Б) с использованием парной корреляционной функции (рис. 4a, б). Обнаружено, что на уровне значимости 0.05 и та, и другая модель не противоречат натурным данным, так как эмпирическая парная корреляционная функция полностью находится вне областей отклонения гипотез.

\section{Обсуждение}

Поскольку модели однородного и неоднородного точечного поля Пуассона, не учитывающие взаимодействие насечек, после тестирования были признаны не соответствующими наблюдаемым данным (рис. 2a, б), то «расталкивающее взаимодействие» между насечками, т.е. прогнозирование самками усача личиночной конкуренции, можно признать существующим. Однако на данном этапе исследований предположение о «положительном взаимодействии», т.е. учете самками кооперации будущих личинок, способной ослабить устойчивость дерева, также не может быть отвергнуто, так как модель с «кооперацией» не отклонена.

Ранее было показано, что похожее поведение демонстрируют и другие насекомыефитофаги. Самки тополевой моли, личинки которой питаются тканью листа, не выходя на его поверхность, образуя «мину» с центром в месте прикрепления яйца, также оптимизируют размещение потомства. Распределение яиц моли на листе тополя уже на стадии их откладки проявляет черты «расталкивания» с расстоянием взаимодействия, обеспечивающим необходимую площадь питания (Секретенко и др., 2002). Стратегия преодоления антибиозной реакции листа дерева выражается при этом в образовании групп яиц с сохранением «расталкивания» внутри групп.

Оцененные функции взаимодействия насечек усача равны нулю только на расстоянии до $0.5-1$ см, хотя для питания личинки нужна площадка с радиусом около 4 см. К такому расхождению может привести целый ряд причин. Может быть, ущерб от частичного перекрытия зон питания компенсируется преимуществами от совместного преодоления энтоморезистентности дерева. Возможно, жуки оценивают по- 
ложение насечек с некоторыми ошибками. К тому же когда на пике вспышки массового размножения самок много, а незанятых пригодных для заселения стволов мало, то, возможно, требования к величине незанятой площади снижаются и следует с увеличением плотности насечек ожидать уменьшения радиуса взаимодействия. Еще одной причиной может быть то, что взаимодействие насечек, вероятно, не является парным, как это предполагалось в данной работе, и при выборе места откладки яйца самка учитывает не только расстояния до других близлежащих насечек, но и общую конфигурацию их расположения.

Другое возможное объяснение состоит в том, что анализировалось расположение насечек, а не самих яиц, координаты которых соответствуют положению насечек только с известной точностью. Во-первых, не каждая насечка содержит яйцо, около $30 \%$ из них пустые, так как оставлены самками из-за угрозы засмоления яиц (Исаев и др., 1988). В нашей работе $11 \%$ от общего числа насечек были заполнены смолой. Это хорошо просматривалось на фотографиях, и такие насечки не использовались в анализе. Возможно, еще какая-то их часть не содержала яиц, создавая тем самым «шум» в рисунке размещения. В то же время известно, что самки усачей прекрасно отличают пустые насечки от занятых (Anbutsu, Togashi, 2000). Во-вторых, яйцо расположено на расстоянии 0.5 см от одной из длинных сторон насечки, и по фотографиям нельзя определить, с какой именно стороны оно находится.

Мы предполагали, что функция взаимодействия одна и та же для всех насечек, независимо от того, на каком дереве они находятся. В анализе использованы деревья разного диаметра, от 12 до 41 см. Учет диаметра дерева, скорее всего, должен привести к зависимости расстояния взаимодействия от диаметра дерева, так как у крупных деревьев слой луба толще и при том же объеме съеденной пищи радиус конкуренции должен быть меньше. В данном исследовании функция взаимодействия также считается не зависящей от локальной густоты. Тем самым предполагается, что ресурс различается не объемом доступного корма, а какими-то другими свойствами, например безопасностью для потомства, хотя учет неоднородного распределения питательных свойств корма мог бы быть полезен.

\section{Заключение}

Применение методов случайных точечных полей для изучения пространственного распределения яиц насекомых на кормовом объекте позволило выявить особенности репродуктивной стратегии у скрытоживущих насекомых. Полученные в данной работе результаты свидетельствуют в пользу того, что у жуков черного пихтового усача существует стратегия откладки яиц, позволяющая уменьшить конкуренцию за корм между будущими личинками за счет уменьшения возможности перекрытия областей питания отдельных личинок. Показано, что насечки, через которые самки усача помещают яйца под кору, располагаются на стволе так, что в ближайшей окрестности у каждой из них, как правило, количество других насечек значимо меньше, чем это было бы при их независимом распределении. Размещение насечек может быть описано моделью Гиббса с функцией взаимодействия, характеризующей постепенное уменьшение интенсивности «расталкивания» с расстоянием. Критическая величина расстояния между насечками, при достижении которой зависимость в их расположении становится несущественной, составляет около 4 см, что примерно согласуется с размером площади питания, необходимой для выживания одной личинки. Вопрос об учете жуками усача в про- 
цессе откладки яиц кооперативного эффекта, связанного с коллективным преодолением насекомыми защитной реакции дерева на уровне отдельных сравнительно небольших участков ствола, остался открытым и нуждается в дальнейшем исследовании.

\section{Приложение А}

\section{Случайные точечные поля}

Случайные точечные поля, или процессы, являются моделями для описания дискретных множеств точек, случайно распределенных в пространстве (Амбарцумян и др., 1989). В данной работе рассматривается только двумерное пространство, т.е. плоскость. Понятие «пространственный точечный процесс», или точечное поле, не предполагает изменений во времени, а представляет собой идеализированное описание природного механизма, приводящего к ситуации, когда дискретные объекты рассеяны во множестве на значительной площади (Stoyan, Stoyan, 1994; Perry et al., 2006).

Одной из важных характеристик точечного поля является его интенсивность. «Интенсивностью (или плотностью) поля $\lambda$ называется среднее число точек, попадающих в единицу площади» (Вентцель, Овчаров, 1983, c. 79). Интенсивность может быть постоянной: $\lambda=$ conts, тогда поле называется однороднылм, или зависеть от координат: $\lambda=\lambda(x)$, в этом случае поле называется неоднородным. Вероятность обнаружить точку поля в малой области площадью $d x$ с центром в точке плоскости $x$ равна $\lambda(x) d x$. Другим свойством является изотропность, означающая, что характеристики точечного поля не зависят от направления.

Точки поля могут взаимодействовать или не взаимодействовать между собой. Поля без взаимодействия между точками обладают тем свойством, что «вероятность попадания того или иного числа точек в любую область плоскости не зависит от того, сколько их попало в любую другую область, не пересекающуюся с данной» (Вентцель, Овчаров, 1983, с. 79). Пуассоновские точечные поля - это поля без взаимодействия между точками, обладающие еще тем свойством, что в одной и той же точке плоскости практически невозможно встретить две или более точки поля. Пуассоновское точечное поле может быть однородным или неоднородным в соответствии с тем, постоянна или нет его интенсивность. Однородное пуассоновское поле характеризует «полную пространственную случайность», когда не только нет взаимодействия между точками поля, но и его интенсивность постоянна.

Взаимодействие двух произвольных точек поля описывается интенсивностью второго порядка $\rho^{(2)}(x, u)$ так, что вероятность обнаружить одну из них в малой области площадью $d x$ с центром в координатах $x$ при условии, что другая находится в области площадью $d u$ с центром в $u$, задается величиной $\rho^{(2)}(x, u) d x d u$ (Stoyan, Stoyan, 1994; Moller, Waagepetersen, 2007, c. 648). Для неоднородного изотропного поля, если характеристики взаимодействия его точек одинаковы во всех областях плоскости, $\rho^{(2)}(x, u)=\lambda(x) \cdot \lambda(u) \cdot g(r)$, где $\lambda(x), \lambda(u)$ - интенсивность первого порядка в соответствующих точках плоскости, $r$ - расстояние между ними, $g(r)$ - функция, описывающая изменение интенсивности второго порядка в зависимости от этого расстояния и называющаяся парной коррелячионной функuฺией. Для неоднородного изотропного поля $g(r)=\frac{\rho^{(2)}(x, u)}{\lambda(x) \cdot \lambda(u)}$. Для однородного изотропного поля $g(r)=\frac{\rho^{(2)}(r)}{\lambda^{2}}$. Если точки не взаимодействуют, $g(r)=1$ при любых расстояниях. Для однородных и изотропных полей парная 
корреляционная функция характеризует, как изменяется с расстоянием от произвольно выбранной точки поля частота встречаемости других точек поля. Если точки поля образуют скопления в результате «притяжения», значения парной корреляционной функции больше единицы на расстояниях, сопоставимых с размером скоплений. При регулярном размещении, или отталкивании, парная корреляционная функция меньше единицы от нуля до расстояния, на котором взаимодействие между точками исчезает.

Для описания систем взаимодействующих точечных объектов существует множество моделей, учитывающих разные варианты взаимодействия точек между собой (Stoyan, Stoyan, 1994; Møller, Waagepetersen, 2007; Gelfand et al., 2010). Распространенным инструментом моделирования взаимодействия типа «расталкивания» являются гиббсовские точечные поля (Ripley, 1990; Stoyan, Stoyan, 1994; Грабарник, 2007; Грабарник, Щербаков, 2012). Эти модели возникли в статистической физике для описания систем взаимодействующих частиц. Особенность точечных полей Гиббса заключается в том, что вместо функции интенсивности используется функция условной интенсивности $\lambda(u, \boldsymbol{X})$. Эта функция задает вероятность $\lambda(u, \boldsymbol{X}) d u$ обнаружить точку поля в малой области площадью $d u$ с центром в точке плоскости с координатами $u$, при условии, что положение всех остальных точек поля уже известно и задано координатами $\boldsymbol{X}$. Если взаимодействие точек поля парное, т.е. взаимодействие двух точек не меняется с появлением третьей, четвертой и т.д. точек, то условная интенсивность неоднородного гиббсовского поля в каждой точке плоскости задается в виде произведения функций

$$
\lambda(u ; \mathbf{X})=b(u) \prod_{\substack{i=1 \\ x_{i} \neq u}}^{n} h\left(u, x_{i}\right),
$$

где $u$ - координата точки плоскости; $\boldsymbol{X}$ - вектор координат точек поля; $b(u)$ - функция, описывающая детерминированную зависимость условной интенсивности от координаты плоскости $u ; h\left(u, x_{i}\right)$ - функция взаимодействия точек поля, отражающая стохастическую компоненту, зависящая от координаты плоскости $u$ и от координат точки поля $x_{i} ; n-$ число точек поля. В случае однородного поля Гиббса функция $b(u)$ не зависит от координат: $b(u)=b$. В изотропном случае взаимодействие зависит только от расстояния между точками поля $r$. Значения $h(r)$ больше единицы соответствуют «притяжению», меньше единицы-«расталкиванию» точек поля. Функция взаимодействия обычно задается так, чтобы обозначить, что за пределами некоторого расстояния между двумя точками взаимодействия между ними нет. Например, функция Штрауса описывает взаимодействие так, что оно не меняется на расстояниях от нуля до $R(c-$ константа) и отсутствует на расстояниях больше $R$ :

$$
h(r)=\left\{\begin{array}{ll}
c & \text { если } r \leq R \\
1 & \text { если } r>R
\end{array} .\right.
$$

\section{Приложение Б}

\section{Методы анализа точечных структур}

Результат наблюдения природного явления, которое может быть описано с помощью модели случайного точечного поля, - набор точек, соответствующих координатам объектов в пределах учетной площадки. Реализация модели точечного поля в ограниченной области - набор точек, заданных координатами. Вариантов реализаций одной и той же модели может быть сколько угодно много. Данные наблюдений и реализации моделей, называемые конфигурациями точек, являются конечными множествами точек $\mathbf{X}=\left\{x_{i} \in A, i=1, \ldots, n\right\}$, заданных своими координатами в области $A$. Здесь $n$ - число точек конфигурации; $x_{i}$ для точки 
конфигурации с номером $i$ в случае размещения на плоскости обозначает для краткости сразу две координаты по обеим осям (Stoyan, Stoyan, 1994; Грабарник, Щербаков, 2012).

В данной работе расчеты выполнены в статистической среде R (R Core Team, 2013), в основном с использованием пакета spatstat, предназначенного для исследования точечных структур (Baddeley, Turner, 2005).

\section{Описательные характеристики} точечных конфигурачий

Функция интенсивности $\hat{\lambda}(u, h)$ в любой точке учетной площадки с координатами $u$ может быть задана численно как количество точек конфигурации, попавшее в круг радиуса $h$ с центром в точке $u$ в расчете на единицу его площади. Для того чтобы близко расположенные точки конфигурации больше влияли на $\hat{\lambda}(u, h)$, чем точки, лежащие в отдалении, используются взвешенные методы с убывающими по расстоянию функциями, задающими вклад точек. В пакете spatstat для такой оценки функции интенсивности применяется взвешенное сглаживание с ядром в виде функции Гаусса (Gelfand et al., 2010).

Выборочная парная коррелячионная функиия для однородного изотропного точечного поля в самом простом случае рассчитывается в два шага. Сначала получают оценку плотности второго порядка, которая описывает, как меняется с расстоянием от типичной точки конфигурации плотность других точек конфигурации. Затем полученную функцию делят на оценку средней по учетной площадке интенсивности. В пакете spatstat pacсчитывается более сложный вариант - сглаженная эмпирическая парная корреляционная функция с коррекцией граничного эффекта. В случае неоднородного поля в процессе расчета выполняется нормировка с использованием предварительно оцененной функции интен- сивности, отражающей изменение интенсивности по площади (Baddeley, Turner, 2005; Gelfand et al., 2010).

Оиенивание параметров моделей по данным наблюдений

Однородное пуассоновское поле имеет единственный параметр - интенсивность $\lambda$, который оценивается как $\hat{\lambda}=n / A$, где $n$ - число точек паттерна, $A$ - величина площади наблюдения. Неоднородное пуассоновское поле задается функиией интенсивности $\hat{\lambda}(u)$, оцененной в данной работе непараметрическим методом, описанным выше.

Для оценивания параметров моделей точечных полей с взаимодействием в пакете spatstat имеется специально разработанный вычислительный алгоритм, включающий численную максимизацию функции псевдоправдоподобия (Baddeley, Turner, 2005). В общем виде функция псевдоправдоподобия $P L(\theta ; \mathbf{X})$ для модели с взаимодействием записывается так:

$$
P L(\theta ; \mathbf{X})=\left(\prod_{i=1}^{n} \lambda_{\theta}\left(x_{i} ; \mathbf{X}\right)\right) \exp \left(-\int_{A} \lambda_{\theta}(u ; \mathbf{X}) d u\right) .
$$

Оцененными параметрами модели становятся такие значения вектора $\theta$, при которых $P L(\theta ; \mathbf{X})$ достигает максимума (Baddeley, Turner, 2000). Функция взаимодействия после оценивания параметров модели по натурным данным дает информацию о направленности и силе взаимодействия элементов точечного поля между собой.

Проверка статистических гипотез согласия моделей точечных полей с данными

Проверку согласия моделей точечных полей и натурных данных выполняли методом статистического моделирования путем многократных имитаций точечных размеще- 
ний, соответствующих моделям. Параметры моделей предварительно оценивали на предыдущем этапе исследования. Для каждой реализации модели рассчитывали парную корреляционную функцию и затем по всей их совокупности определяли границы двусторонней области отклонения гипотезы.

Для моделирования однородного пуассоновского поля в соответствии с заданной интенсивностью $\hat{\lambda}$ вначале генерируется величина $N_{s,}$ - общее число точек конфигурации, распределенное по закону Пуассона с параметром $\hat{\lambda} S$, где $S$ - площадь учетной площадки. Затем последовательно независимо генерируется $N_{s}$ точек конфигурации, каждая с координатами, равномерно распределенными в пределах учетной площадки.

Неоднородное пуассоновское поле моделируется методом исключения фон Неймана (Михайлов, 1975). Предварительно в изучаемой области $S$ по данным наблюдений оценивается зависящая от координат интенсивность точечного поля $\hat{\lambda}(u)$. Затем генерируется однородный вариант поля с интенсивностью $\hat{\lambda}_{\max }=\max _{u \in S} \hat{\lambda}(u)$, после чего каждая сгенерированная точка с координатами $u$ удаляется с вероятностью $1-\hat{\lambda}(u) / \hat{\lambda}_{\max }$ (Грабарник, 2007).

Распределение Гиббса имитируется с помощью алгоритма Метрополиса-Хастингса (Грабарник, 2007). Для этого предварительно генерируется, например, реализация пуассоновского поля с необходимым количеством точек. Затем многократно повторяется процедура, суть которой сводится к следующему. Случайно выбранная точка конфигурации перемещается в новую случайно выбранную точку выборочной площадки. После этого рассчитывается величина, называемая «полной энергией системы», зависящая от функции взаимодействия (имеющей параметры, оцененные на предыдущем шаге анализа) и текущего расположения всех точек конфигу- рации. Перемещенная точка может остаться на новом месте или вернуться на старое: если полная энергия системы в результате перемещения уменьшилась, то она с большей вероятностью окончательно занимает новое место. Эта процедура выполняется до тех пор, пока полная энергия конфигурации точек не перестанет меняться. Имитация неоднородного гиббсовского поля включает учет детерминированной компоненты, заданной численно или аналитически в виде двумерной функции от координат плоскости. Описанные алгоритмы, реализованные в пакете spatstat, использованы в нашей работе для тестирования гипотез методом Монте-Карло.

Метод огибающих. Генерируется $K$ реализаций модели, и для каждой из них рассчитывается эмпирическая парная корреляционная функция $\hat{g}\left(r_{t}\right)$. Значения всех парных корреляционных функций для каждой конкретной величины $r$ ранжируются, после чего проводятся верхняя и нижняя огибающие так, что за их пределами, сверху и снизу, остается по $k$ значений для каждого $r$. Когда в качестве тестовой статистики выбрано значение $\hat{g}\left(r_{t}\right)$ при каком-либо конкретном расстоянии $r_{t}$, то гипотеза согласия модели и данных отклоняется при уровне значимости $\alpha=k / K$ в том случае, если значение парной корреляционной функции $\hat{g}\left(r_{t}\right)$, полученной по натурным данным, выходит за пределы огибающих (Stoyan, Stoyan, 1994). В настоящей работе для тестирования использовался диапазон расстояний $0.5-12 \mathrm{~cm}$ одновременно. В таком случае вероятность ошибки первого рода может быть определена как $1-k_{0} / K$, где $k_{0}$ - число тех реализаций модели, парные корреляционные функции которых целиком лежат между огибающими (Grabarnik et al., 2011). Для представленных в настоящей работе данных после некоторого количества пробных расчетов установлено, 
что для всех тестируемых моделей величины $K=1500, k=3$ приводят к вероятности ошиб- ки первого рода от 0.033 до 0.047 , т.е. меньше чем $5 \%$.

\section{Работа поддержана РФФИ (грант 15-04-01192).}

\section{Список литературы}

1. Амбарцумян Р.В., Мекке Й., Штойян Д. (1989) Введение в стохастическую геометрию. М.: Наука, 400 с.

2. Бахвалов С.А., Колтунов Е.В., Мартемьянов В.В. (2010) Факторы и экологические механизмы популяционной динамики лесных насекомых-филлофагов. Новосибирск: Изд-во СО PAH, $299 \mathrm{c}$.

3. Вентцель Е.С., Овчаров Л.А. (1983) Прикладные задачи теории вероятности. М.: Радио и связь, 416 с.

4. Грабарник П.Я. (2007) Моделирование пространственной структуры древостоев. В: Кудеяров В.Н. (отв. ред.) Моделирование динамики органического вещества в лесных экосистемах. М.: Наука, с. 132-146.

5. Грабарник П.Я. (2010) Анализ горизонтальной структуры древостоя: модельный подход. Лесоведение 2: 77-85.

6. Грабарник П.Я., Щербаков В.В. (2012) О модели точечных конфигураций, заданной полупараметрическим взаимодействием. Вестник Московского университета. Серия 1: Математика. Механика 2: 3-8.

7. Исаев А.С., Гирс Г.И. (1975) Взаимодействие дерева и насекомых-ксилофагов. Новосибирск: Наука, 346 с.

8. Исаев А.С., Рожков А.С., Киселев В.В. (1988) Черный пихтовый усач Monochamus urussovi (Fisch.). Новосибирск: Наука, 272 с.

9. Исаев А.С., Хлебопрос Р.Г., Недорезов Л.В., Кондаков Ю.П., Киселев В.В., Суховольский В.Г. (2001) Популяционная динамика лесных насекомых. М.: Наука, 374 с.

10. Кокс Д., Льюис П. (1969) Статистический анализ последовательностей событий. М.: Мир, 312 с.

11. Кузьмичев В.В., Секретенко О.П. (2001) Связь горизонтальной структуры и динамики состава сосново-лиственничных культур. Лесоведение 5: 81-88.

12. Курбанов Э.А., Воробьев О.Н. (2008) Пространственное распределение крупного древесного детрита в сосняках марийского Заволжья. Экология 2: 103-109.

13. Михайлов Г.А. (1975) Некоторые вопросы теории методов Монте-Карло. Новосибирск: Наука, 143 с.

14. Мозолевская Е.Г., Селиховкин А.В., Ижевский С.С. (2010) Лесная энтомология. М.: Академия, $416 \mathrm{c.}$

15. Павлов С.И. (2011) Этолого-экологические адаптации в процессе проявления превентивной заботы о потомстве у жуков-листоедов (Coleoptera,Cchrysomelidae). Вестник Волжского университета им. В.Н. Татищева 12: 72-78.

16. Секретенко О.П., Суховольский В.Г., Тарасова О.В. (2002) Оптимизационная модель пространственной структуры популяции (на примере размещения яиц тополевой моли на листе). Журнал общей биологии 4: 351-360. 
17. Ухваткина О.Н., Омелько А.М. (2011) Структура подроста и естественное возобновление в смешанном хвойно-широколиственном лесу Южного Сихотэ-Алиня. Журнал Сибирского федерального университета. Серия: Биология 3: 266-280.

18. Фардеева М.Б., Чижикова Н.А., Бирючевская Н.В., Рогова Т.В., Савельев А.А. (2009) Математические подходы к анализу пространственно-возрастной структуры популяций дерновинных видов трав. Экология 4: 249-257.

19. Anbutsu H., Togashi K. (1997) Effects of spatio-temporal intervals between newly-hatched larvae on larval survival and development in Monochamus alternatus (Coleoptera: Cerambycidae). Researches on Population Ecology 39(2): 181-189.

20. Anbutsu H., Togashi K. (2000) Deterred oviposition response of Monochamus alternatus (Coleoptera: Cerambycidae) to oviposition scars occupied by eggs. Agricultural and Forest Entomology 3: 217-223.

21. Arimura G., Huber D.P.W., Bohlmann J. (2004) Forest tent caterpillars (Malacosoma disstria) induce local and systemic diurnal emissions of terpenoid volatiles in hybrid poplar (Populus trichocarpa $\mathrm{x}$ deltoides): cDNA cloning, functional characterization, and patterns of gene expression of (-)-germacrene D synthase, PtdTPS1. The Plant Journal 37: 603-616.

22. Baddeley A., Turner R. (2000) Practical maximum pseudolikelihood for spatial point patterns (with discussion). Australian and New Zealand Journal of Statistics 42(3): 283-322.

23. Baddeley A., Turner R. (2005) Spatstat: an R package for analyzing spatial point patterns. Journal of Statistical Software 12(6): 1-42.

24. Baddeley A., Gregori P., Mateu J., Stoica R., Stoyan D. (eds) (2006) Case studies in spatial point process modeling. Lecture notes in statistics 185. N.Y.: Springer, $306 \mathrm{p}$.

25. Castillo D.M., Kula A.A.R., Fenster K.A.D., Fenster C.B., Dudash M.R. (2013) Specialist pollinating seed predator exhibits oviposition strategy consistent with optimal oviposition theory. Ecological Entomology 38: 164-172.

26. Clark B.D., Faeth S.H. (1997) The consequences of larval aggregation in the butterfly Chlosyne Iacinia. Ecological Entomology 22: 408-415.

27. Denno R.F., Benrey B. (1997) Aggregation facilitates larval growth in the neotropical nimphalid butterfly Chlosyne janais. Ecological Entomology 22: 133-141.

28. Desurmont G.A., Weston P.A. (2011) Aggregative oviposition of a phytophagous beetle overcomes egg-crushing plant defences. Ecological Entomology 3: 335-343.

29. Gelfand A.E., Diggle P.J., Fuentes M., Guttorp P. (eds) (2010) Handbook of spatial statistics. Boca Raton FL: CRC Press, 619 p.

30. Grabarnik P., Myllymäki M., Stoyan D. (2011) Correct testing of mark independence for marked point patterns. Ecological Modelling 222: 3888-3894.

31. Grohmann C., Oldeland J., Stoyan D., Linsenmair K. E. (2010) Multi-scale pattern analysis of a mound-building termite species. Insectes Sociaux 57: 477-486.

32. Harkness R.D., Isham V.A. (1983) Bivariate spatial point pattern of ants' nests. Journal of the Royal Statistical Society. Series C (Applied Statistics) 3: 293-303.

33. Herms D., Mattson W.J. (1992) The dilemma of plants: to growth or defend. The Quarterly Review of Biology 67(3): 283-335. 
34. Holland J.N., Buchanan A.L., Loubeau R. (2004) Oviposition choice and larval survival of an obligately pollinating granivorous moth. Evolutionary Ecology Research 6: 607-618.

35. Horn K.C., Holland J.M. (2010) Discrimination among floral resources by an obligately pollinating seed-eating moth: host-marking signals and pollination and florivory cues. Evolutionary Ecology Research 12: 119-129.

36. Janz N. (2002) Evolutionary ecology of oviposition strategies In: Hilker M., Meiners T. (eds) Chemoecology of insect eggs and egg deposition. Berlin: Blackwell, p. 349-376.

37. Lempa K., Agrawal A.A., Salminen J.P., Turunen T., Ossipov V., Ossipova S., Haukioja E., Pihlaja K. (2004) Rapid herbivore-induced changes in mountain birch phenolics and nutritive compounds and their effects on performance of the major defoliator, Epirrita autumnata. Journal of Chemical Ecology 30(2): 303-321.

38. Leyva K.J., Clancy K.M., Price P.W. (2003) Oviposition strategies employed by the western spruce budworm: tests of predictions from the phylogenetic constraints hypothesis. Agricultural and Forest Entomology 5(1): 9-16.

39. Møller J., Waagepetersen R.P. (2007) Modern statistics for spatial point processes. Scandinavian Journal of Statistics 4: 643-684.

40. Paukku S., Kotiaho J.S. (2008) Female oviposition decisions and their impact on progeny lifehistory traits. Journal of Insect Behavior 21: 505-520.

41. Peddle S., De Groot P., Smith S. (2002) Oviposition behaviour and response of Monochamus scutellatus (Coleoptera: Cerambycidae) to conspecific eggs and larvae. Agricultural and Forest Entomology 3: 217-222.

42. Perry G.L.W., Miller B.P., Enright N.J. (2006) A comparison of methods for the statistical analysis of spatial point patterns in plant ecology. Plant Ecology 187(1): 59-82.

43. Randlkofer B., Obermaier E., Meiners T. (2007) Mother's choice of the oviposition site: balancing risk of egg parasitism and need of food supply for the progeny with an infochemical shelter? Chemoecology 17(3): 177-186.

44. R Core Team (2013) R: a language and environment for statistical computing. R Foundation for Statistical Computing, Vienna. URL http://www.R-project.org.

45. Ripley B. (1990) Gibbsian interaction models. In: Griffiths D. (ed.) Spatial Statistics: Past, Present and Future. New York: Image, p. 3-28.

46. Rossi J.-P., Samalens J.-C., Guyon D., van Halder I., Jactel H., Menassieu P., Piou D. (2009) Multiscale spatial variation of the bark beetle Ips sexdentatus damage in a pine plantation forest (Landes de Gascogne, Southwestern France). Forest Ecology and Management 257: $1551-1557$.

47. Sadek M.M., Hansson B.S., Anderson P. (2010) Does risk of egg parasitism affect choice of oviposition sites by a moth? A field and laboratory study. Basic and Applied Ecology 11: 135-143.

48. Sekretenko O.P., Gavrikov V.L (1998) Characterization of the tree spatial distribution in small plots using the pair correlation function. Forest Ecology and Management 102: 113-120.

49. Stoyan D., Stoyan H. (1994) Fractals, random shapes and point fields. Chichester: Wiley, 389 p.

50. Uria-Diez J., Ibáñez R., Mateu J. (2013) Importance of habitat heterogeneity and biotic processes in the spatial distribution of a riparian herb (Carex remota $\mathrm{L}$.): a point process approach. Stochastic Environmental Research and Risk Assessment 27: 59-76. 